\title{
Quantitative Structure-Mutation-Activity Relationship Tests (QSMART) model for protein kinase inhibitor response prediction
}

\author{
Liang-Chin Huang ${ }^{1}$, Wayland Yeung ${ }^{1}$, Ye Wang ${ }^{2}$, Huimin Cheng ${ }^{2}$, Aarya Venkat ${ }^{3}$, Sheng Li ${ }^{4}$, Ping Ma ${ }^{2}$, \\ Khaled Rasheed ${ }^{4}$ and Natarajan Kannan ${ }^{1,3^{*}}$
}

*Correspondence:

nkannan@uga.edu

${ }^{1}$ Institute of Bioinformatics,

University of Georgia, 120

Green St., Athens, GA 30602, USA

Full list of author information is available at the end of the article

\begin{abstract}
Background: Protein kinases are a large family of druggable proteins that are genomically and proteomically altered in many human cancers. Kinase-targeted drugs are emerging as promising avenues for personalized medicine because of the differential response shown by altered kinases to drug treatment in patients and cell-based assays. However, an incomplete understanding of the relationships connecting genome, proteome and drug sensitivity profiles present a major bottleneck in targeting kinases for personalized medicine.

Results: In this study, we propose a multi-component Quantitative Structure-Mutation-Activity Relationship Tests (QSMART) model and neural networks framework for providing explainable models of protein kinase inhibition and drug response $\left(\mathrm{IC}_{50}\right)$ profiles in cell lines. Using non-small cell lung cancer as a case study, we show that interaction terms that capture associations between drugs, pathways, and mutant kinases quantitatively contribute to the response of two EGFR inhibitors (afatinib and lapatinib). In particular, protein-protein interactions associated with the JNK apoptotic pathway, associations between lung development and axon extension, and interaction terms connecting drug substructures and the volume/charge of mutant residues at specific structural locations contribute significantly to the observed $I C_{50}$ values in cellbased assays.

Conclusions: By integrating multi-omics data in the QSMART model, we not only predict drug responses in cancer cell lines with high accuracy but also identify features and explainable interaction terms contributing to the accuracy. Although we have tested our multi-component explainable framework on protein kinase inhibitors, it can be extended across the proteome to investigate the complex relationships connecting genotypes and drug sensitivity profiles.
\end{abstract}

Keywords: Protein kinase inhibitor, Precision medicine, Machine learning, Systems pharmacology author(s) and the source, provide a link to the Creative Commons licence, and indicate if changes were made. The images or other third party material in this article are included in the article's Creative Commons licence, unless indicated otherwise in a credit line to the material. If material is not included in the article's Creative Commons licence and your intended use is not permitted by statutory regulation or exceeds the permitted use, you will need to obtain permission directly from the copyright holder. To view a copy of this licence, visit http:// creativecommons.org/licenses/by/4.0/. The Creative Commons Public Domain Dedication waiver (http://creativecommons.org/publi cdomain/zero/1.0/) applies to the data made available in this article, unless otherwise stated in a credit line to the data. 


\section{Background}

Chemotherapy has served as standard care for cancer treatments for decades; however, the resistance of cancer cells to chemotherapy presents a major challenge in effectively treating cancer patients [1]. A major contributing factor in drug resistance [2], as well as drug sensitivity [3], is the accumulation of mutations in oncogenic proteins such as protein kinases, which are primary targets for cancer drugs [4]. Mutations in protein kinases can have varying impacts on drug sensitivity depending on the structural location of mutations. For example, non-small cell lung cancer (NSCLC) cells harboring the T790M mutations in the Epidermal Growth Factor Receptor (EGFR) are resistant to the cancer drug, gefitinib, whereas cells harboring the L858R mutation are hypersensitive to the same drug $[5,6]$. In contrast, cells harboring the double mutant (T790M/L858R) are only resistant to gefitinib but not sensitive to it [7]. As mutations impact the efficacy of different cancer drugs, there is a need to incorporate structural knowledge in drug response prediction methods.

To identify molecular and genomic features associated with drug sensitivity and resistance in cancer cells, the Genomics of Drug Sensitivity in Cancer Project (GDSC) [8] recently screened the drug responses of 266 anticancer drugs against $\sim 1000$ human cancer cell lines. Moreover, to broaden the pharmacologic annotation for human cancers, the Cancer Cell Line Encyclopedia (CCLE) [9] provided the pharmacological profiles of 24 drugs across 504 cancer cell lines. By utilizing these datasets, several prediction models were built to pursue a more accurate drug response estimation by different types of approaches, from traditional statistical models, network-based models, machine learning methods, to state-of-the-art neural networks (Table 1).

Despite progress in the development of computational methods for drug response prediction, existing methods do not have the sensitivity to achieve "precision" medicine goals. The prediction performances measured by the coefficient of determination $\left(R^{2}\right)$ are in the range from 0.25 to 0.78 . More recently, deep neural networks (DNN) with multiple hidden layers such as CDRscan [23], tCNNS [35], and MCA [36] have been proposed that achieve $R^{2}$ higher than $0.8\left(R^{2}=0.84,0.83\right.$, and 0.86 , respectively). However, most of the cancer cell line features used in previous studies are based on gene expression profiles and do not explicitly consider associations between drugs and the structural location of mutations (Table 1). Consequently, the molecular mechanisms of drug-protein interactions cannot be inferred from these models. The trade-off between prediction performance and explainability is also an issue for existing methods, such as CDRscan, tCNNS, and MCA, as they do not explicitly reveal the features that contribute to the observed prediction performance. Consequently, the Defense Advanced Research Projects Agency (DARPA) recently launched the Explainable Artificial Intelligence program (XAI) [38] to facilitate building explainable models while maintaining prediction performance.

In recognition of the interest in building explainable AI models, we built the Quantitative Structure-Mutation-Activity Relationship Tests (QSMART) model, which extends the quantitative structure-activity relationship (QSAR) model to capture drug-mutation relationships. Additionally, it identifies the most informative drug and genomic features contributing to drug sensitivity predictions using traditional statistical and feature selection methods (Fig. 1). Although we cannot explain the entire model to humans in 


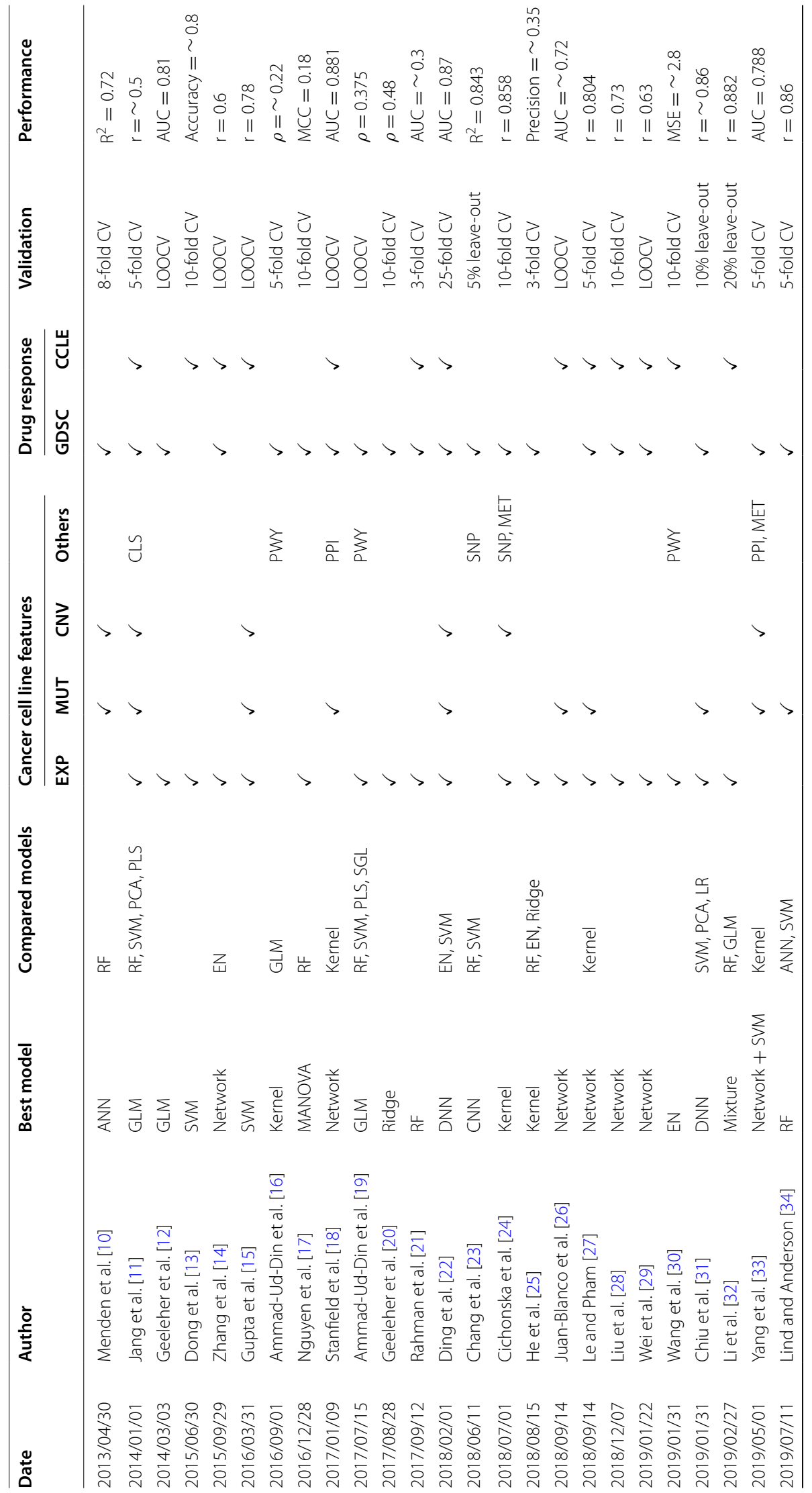




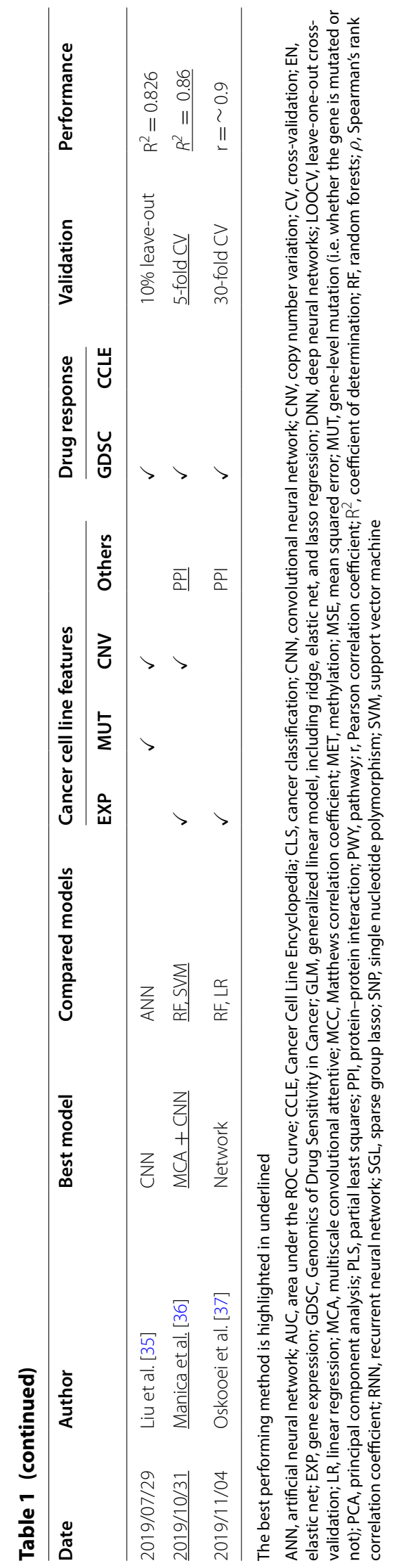




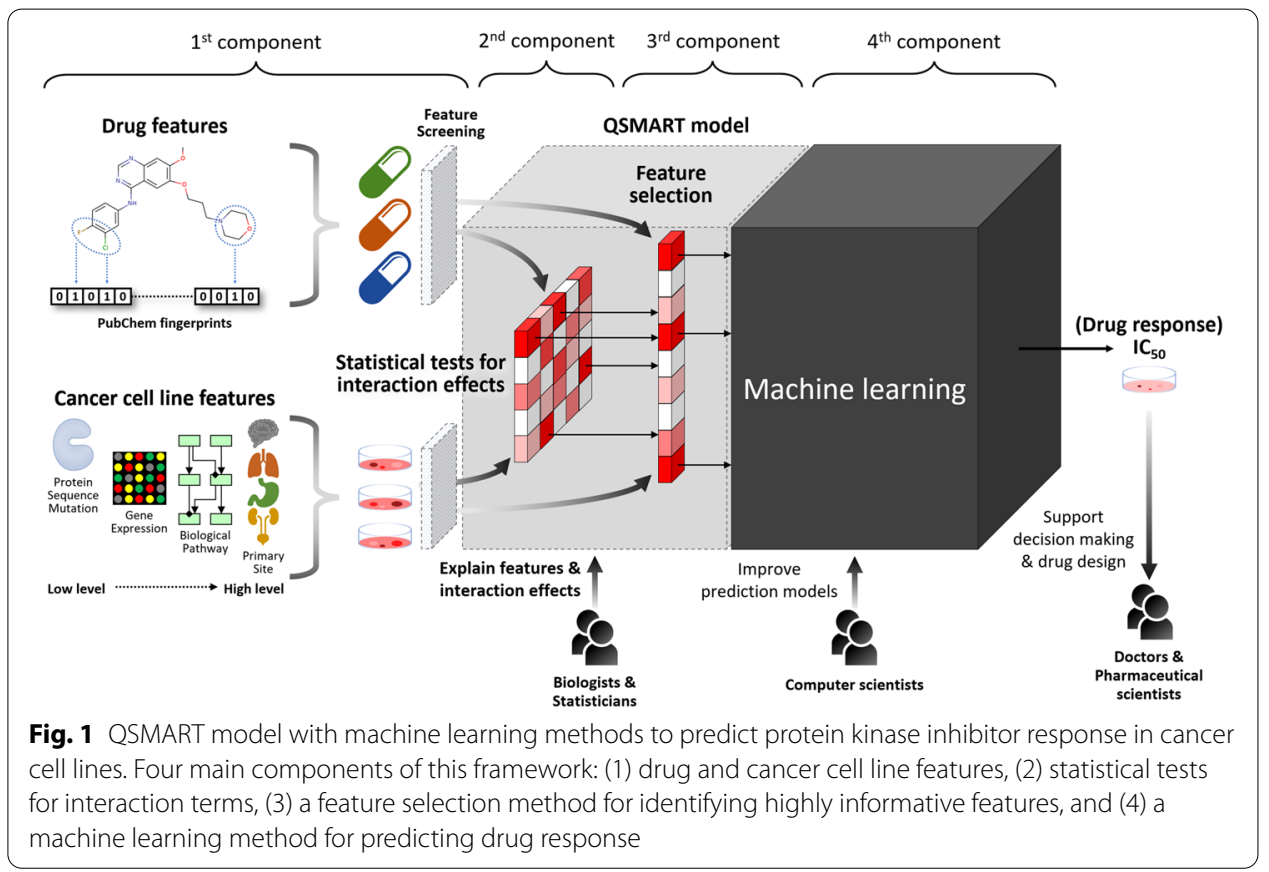

plain language, we show that these steps increase the prediction model's explainability by moving two hidden layers outside the neural networks "black box". The features and interaction terms in these two layers are interpretable by statisticians and biologists. When applied on a subset of protein kinase inhibitors (PKIs), the QSMART model achieves prediction accuracy comparable to or better than the state-of-the-art DNN methods (overall $\mathrm{R}^{2}=0.863$, AUC $=0.981$, and RMSE $=0.811$ ). Our studies represent the first systematic effort to develop explainable models for protein kinase inhibitor response prediction in cancer cell lines.

\section{Results}

\section{Performance of QSMART is comparable to DNN}

The QSMART model with neural networks predicts PKI responses in 23 cancer types with accuracies ranging from $\mathrm{R}^{2}=0.805$ to 0.881 . Figure $2 \mathrm{a}$ presents $\mathrm{IC}_{50}$ versus predicted $\mathrm{IC}_{50}$ plot for all types of cancer cell lines (overall $\mathrm{R}^{2}=0.863$ and $\mathrm{RMSE}=0.811$ ). For each cancer-centric model, Table 2 summarizes the number of PKI responses, the total number of features (including drug features, cancer cell line features, and interaction terms), the number of nodes in the first and second hidden layers of neural networks, and prediction performance $\left(R^{2}\right)$. Additional file 1: Table S1 shows additional measurements of prediction performance (RMSE and AUC), cancer cell line features at seven feature levels, interaction terms, and training iterations. Compared with commonly used machine learning models and a state-of-the-art DNN model, multiscale convolutional attentive (MCA) [36], the QSMART model with neural networks shows higher or comparable performances of predicting PKI response for 23 cancer types based on 10-fold cross-validation (Fig. $2 \mathrm{~b}$ and Table 2). In this study, we designed three types of neural network architectures: single-layer, double-layer, and complex-double-layer. However, we found that the prediction models for all the 23 cancer types can achieve 


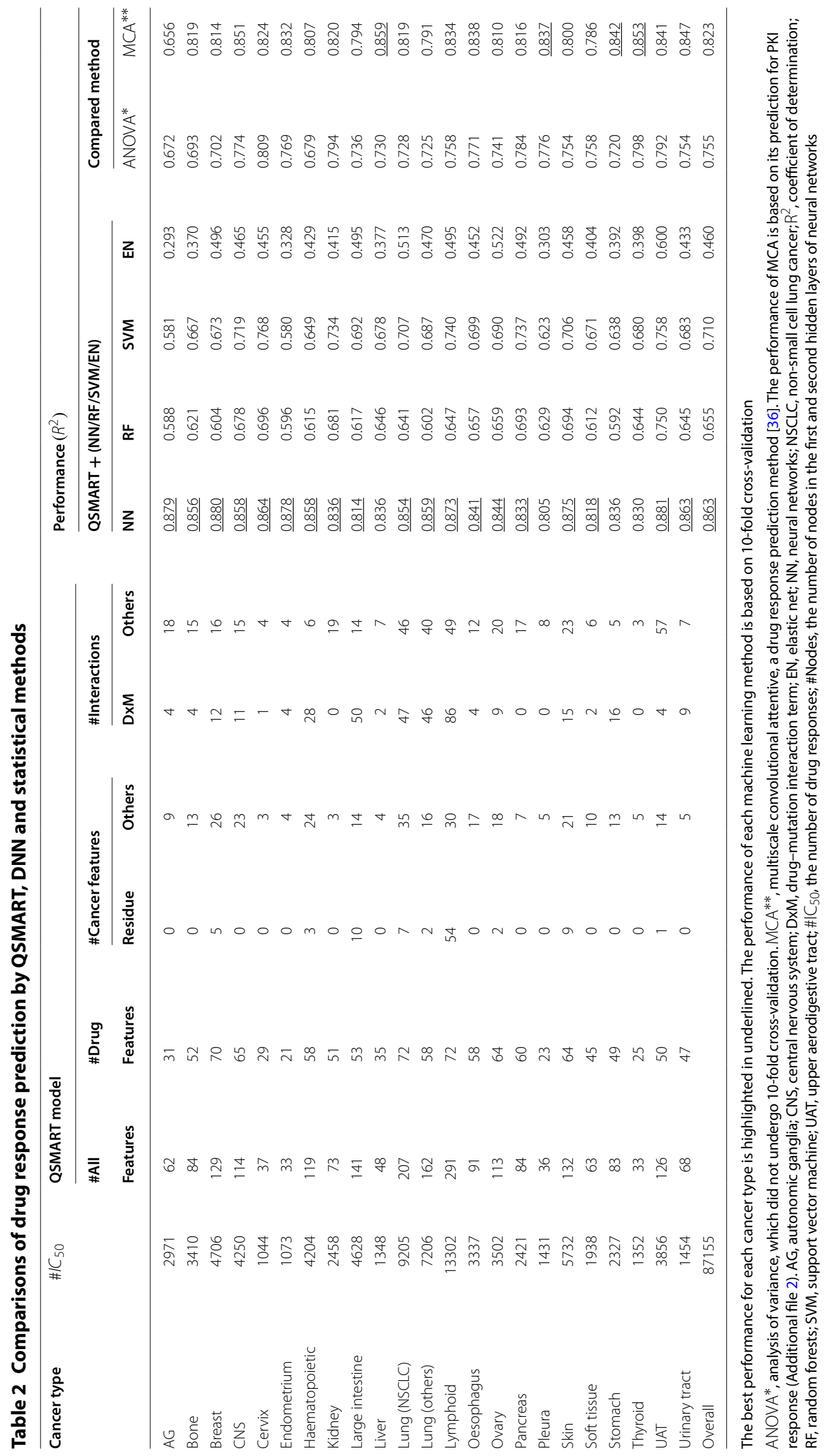



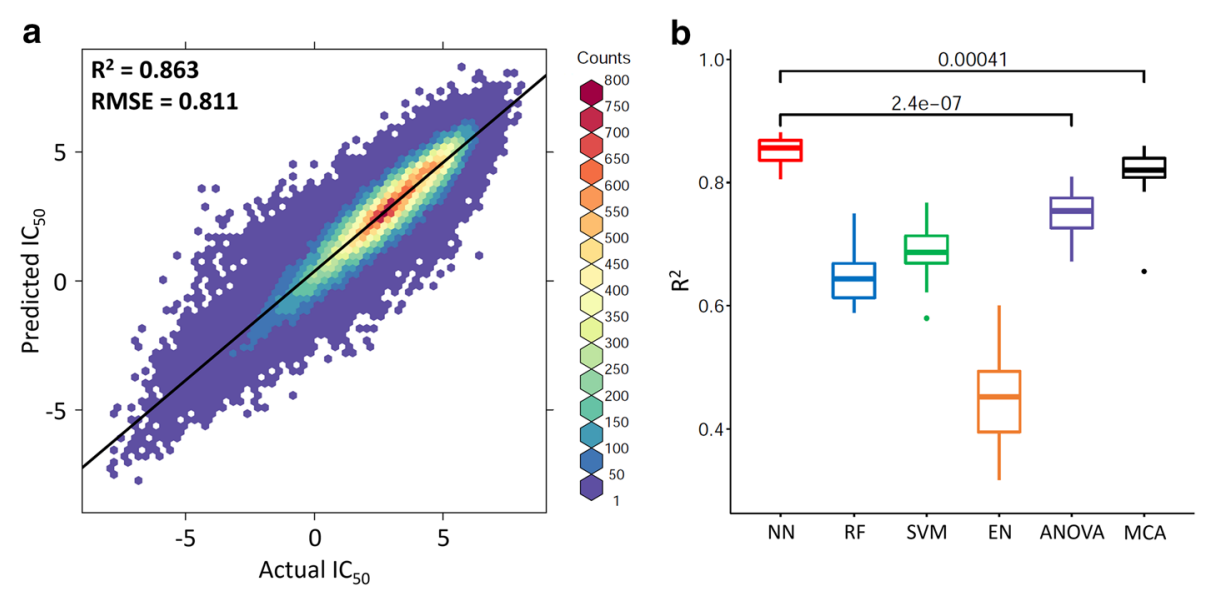

C
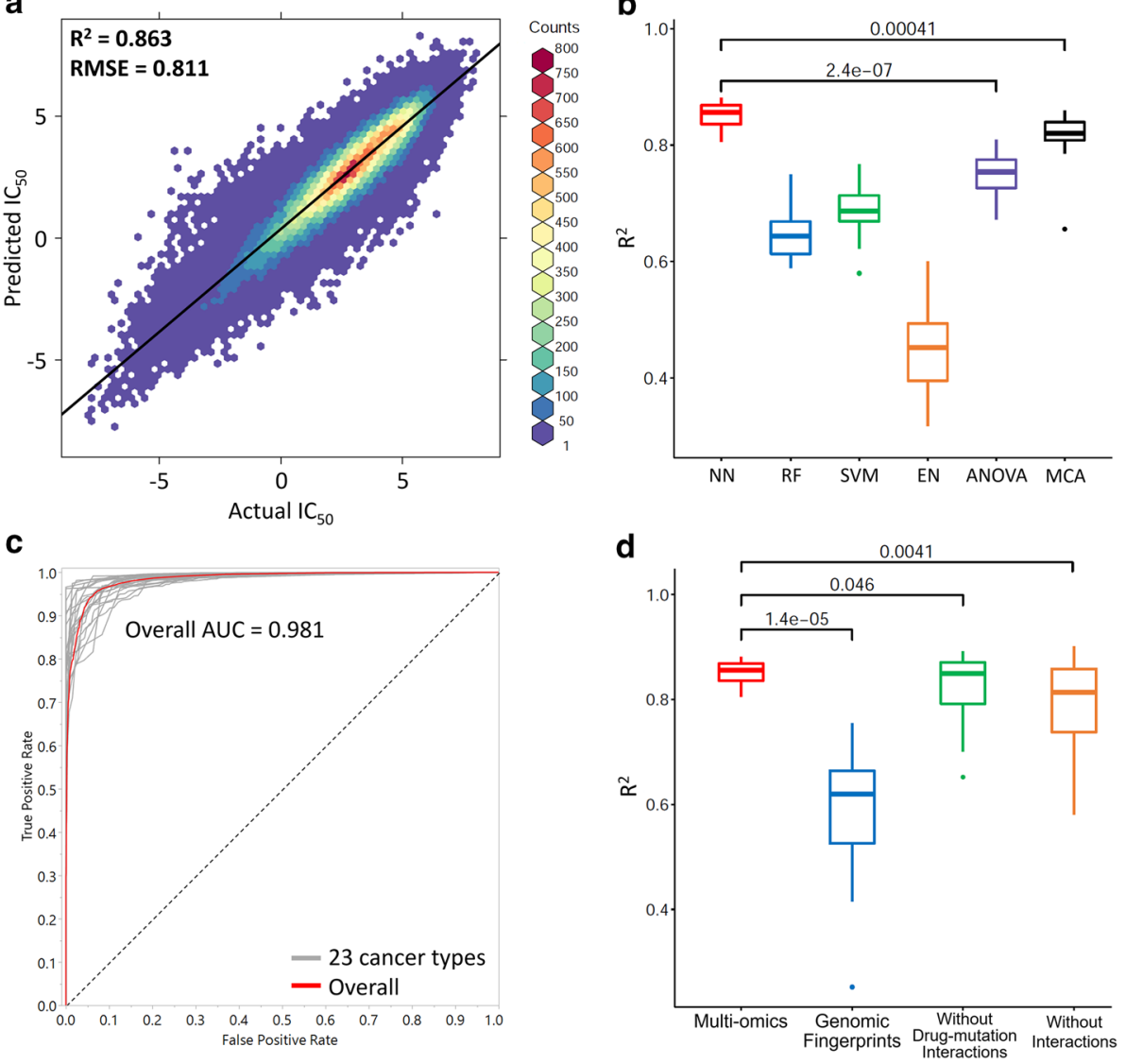

Fig. 2 Prediction performances of different datasets and different prediction models. Wilcoxon signed-rank test is performed, and the $p$ value is shown in each box plot. a Comparison between actual $I C_{50}(x$-axis) and the $I_{50}$ predicted by using QSMART with neural networks across all cancer types (y-axis). A fitted regression line is shown. $\mathbf{b}$ Prediction performances of different statistical or machine learning methods. NN: neural networks; RF: random forests; MCA: multiscale convolutional attentive [36]. c ROC curves for 23 cancer-centric models as well as an overall ROC. $\mathbf{d}$ Impact of different data sets on prediction performance

$\mathrm{R}^{2}>0.8$ by using either single-layer or double-layer architecture. As per Occam's razor principle, we only used the single-layer or double-layer architecture since they are able to achieve accuracies comparable to or better than the state-of-the-art DNN methods.

To further confirm the QSMART model's ability to classify drug responses into two categories (sensitive versus non-sensitive), we chose thresholds to define actual $\mathrm{IC}_{50}$ as sensitive or non-sensitive. Compared to a single threshold used in a previous study [23] $\left(\mathrm{IC}_{50}=-2\right)$, we set multiple thresholds $(-4,-3,-2,-1$, and 0$)$ and averaged the results to avoid overestimating the prediction performance. The resulting ROC curves for 23 cancer types and the overall curve are shown in Fig. 2c. The overall AUC is 0.981 and comparable to a recent DNN-based study [23] (AUC > 0.98). AUC for each cancer type is available in the Additional file 1: Table S1.

\section{Multi-omics data are informative in prediction models}

To investigate the extent to which multi-omics features introduced in this study contribute to drug response prediction, we compared the contribution of multi-omics 
features with simple genomic features such as genomic fingerprints. Genomic fingerprints are binary vectors representing genomic mutation positions. They are the only cancer cell line features used in one of the top-performing methods [23]. Thus we replaced our multi-omics cancer cell line features with 44,364 genomic fingerprints (Additional file 1: Figure S1) and ran our predictions with the same number of features, feature selection methods, and neural network architectures. The number of selected features, including interaction terms, and prediction performances are shown in Additional file 1: Table S2. The box plot in Fig. 2d shows that the performance distribution of 23 cancer-centric models using multi-omics features is significantly higher than that of the models using genomic fingerprints alone (overall $\mathrm{R}^{2}=0.863$ versus $0.655, p$ value $=1.4 \mathrm{e}-05$, Wilcoxon signed-rank test).

\section{Contribution of interaction terms in prediction models}

We next wanted to evaluate the contribution of interaction terms (the second component in Fig. 1) in drug response prediction. We examined the prediction performance by removing drug-mutation interaction terms and removing all interaction terms. We utilized the feature selection method to prioritize all input features, selected the same total number of features in the original models shown in Table 2, and then used the same neural network architectures to train the new models. The results of these two experiments are shown in Additional file 1: Table S3 and Table S4, respectively. The box plot in Fig. 2d shows that the performance of the full QSMART model is better than the models without drug-mutation interaction terms (overall $R^{2}=0.863$ versus $0.846, p$ value $=0.046$ ) and the models without any interaction terms (overall $\mathrm{R}^{2}=0.863$ versus $0.817, p$ value $\left.=0.0041\right)$. Intriguingly, for some cancer types, such as breast, models without any interaction terms achieve better performance than the QSMART model. This is likely because some more informative high-order interactions (three-way or even multi-way interactions), which cannot be detected by the statistical method we used, were captured inside the neural network black box and thus compensated for the lack of interaction terms in the input layer. However, neural networks cannot guarantee that these informative but unexplainable high-order interactions will always be captured under the limited number of samples and the training iteration we used. This fact is reflected in Fig. $2 \mathrm{~d}$, which shows that the prediction performance is variable when the drug-mutation interaction terms are eliminated $\left(R^{2}\right.$ $=0.653$ to 0.892$)$, or all interaction terms are eliminated $\left(R^{2}=0.581\right.$ to 0.901$)$.

\section{Case study: non-small cell lung cancer}

We next evaluated the contribution of different features in drug response prediction using non-small cell lung cancer (NSCLC) as a case study. All 207 features in the NSCLC-specific QSMART model and their descriptions are listed in Additional file 3. We choose several pertinent features and explain their biological relevance in this case study to demonstrate how scientists may use our prediction model to explain their findings. 


\section{Batch effects are significant factors influencing drug response}

We first wanted to evaluate how drug response datasets generated from different sources contribute to drug response prediction. To this end, we introduced a feature termed "From_Sanger" in the model to distinguish the assays done by the Wellcome Sanger Institute (1) from the Massachusetts General Hospital (0). On average, the PKI responses obtained from Massachusetts General Hospital showed lower drug sensitivity (higher $\mathrm{IC}_{50}$ value) than those from the Wellcome Sanger Institute in the NSCLC dataset (average actual $\mathrm{IC}_{50}=2.88$ versus $2.41, p$ value $=1.3 \mathrm{e}-23$, Wilcoxon rank-sum test). To investigate these experimental batch effects, we increased the value of "From_Sanger" by one unit and held other features constant. If we replace 0 with 1 for the "From_Sanger" feature, the average $\mathrm{IC}_{50}$ predicted by the pre-trained model reduces to 0.65 (average predicted $\mathrm{IC}_{50}=2.87$ versus 2.22, Additional file 3). Notably, this feature is selected not only in the NSCLC model but also in the other 22 cancer-centric models, implying that batch effects are significant factors for drug response prediction.

\section{Contribution of Gene Ontology terms in drug response prediction}

Next, we wanted to investigate how biological process interactions can contribute to drug response prediction. A biological process interaction term "GO_0030324_X_ GO_0048675" is selected in the NSCLC model. This feature represents the product of the number of mutations perturbing the biological process "lung development" (Gene Ontology ID: GO:0030324) and the number of mutations perturbing "axon extension" (Gene Ontology ID: GO:0048675). Axon initiation, extension, and guidance are known to play essential roles in cancer invasion and metastasis [39]. In the NSCLC dataset, there are eight cell lines with mutations in protein kinases associated with axon extension; among them, NCI-H1944 and NCI-H2030 are from patients with metastatic NSCLC. On average, the NSCLC cell lines with "GO_0030324_X_GO_0048675" interaction showed higher PKI responses than those without this interaction (average actual $\mathrm{IC}_{50}=4.32$ versus $2.69, p$ value $=1.4 \mathrm{e}-27$, Wilcoxon rank-sum test). Comparatively, the NSCLC cell lines with mutations involved in "lung development" or "axon extension" alone showed lower PKI responses (average actual $\mathrm{IC}_{50}=3.20$ or 2.07 , respectively). Based on our prediction model, every unit increase in the interaction term "GO_0030324_X_GO_0048675" is associated with a 0.45 unit increase in $\mathrm{IC}_{50}$ on average (average predicted $\mathrm{IC}_{50}=2.73$ versus 3.18 ). This suggests that the lower PKI sensitivity for the NSCLC cell lines is likely due to mutations in genes involved in lung development (e.g., PDGFRA) and axon extension pathway (e.g., DCLK1 or ULK2).

\section{Example of how PPIs contribute to drug response}

The NSCLC model contains 27 protein-protein interaction (PPI) terms. We quantify each PPI by the product of the gene expression level of individual proteins in the complex. Every unit of gene expression level increase in these 27 PPIs contributes to -0.089 to 0.061 unit increase in $\mathrm{IC}_{50}$ on average. Gene enrichment analysis of the 27 genes in the TP53-centric subnetwork (shown in Fig. 3) revealed an overrepresentation of pathways associated with angiogenesis, inflammation, apoptosis, and axon guidance (Additional file 1: Table S5, performed by PANTHER [40]). MAP4K4 is one of the genes involved in the apoptosis signaling pathway, and its over-expression is a prognostic factor for lung 


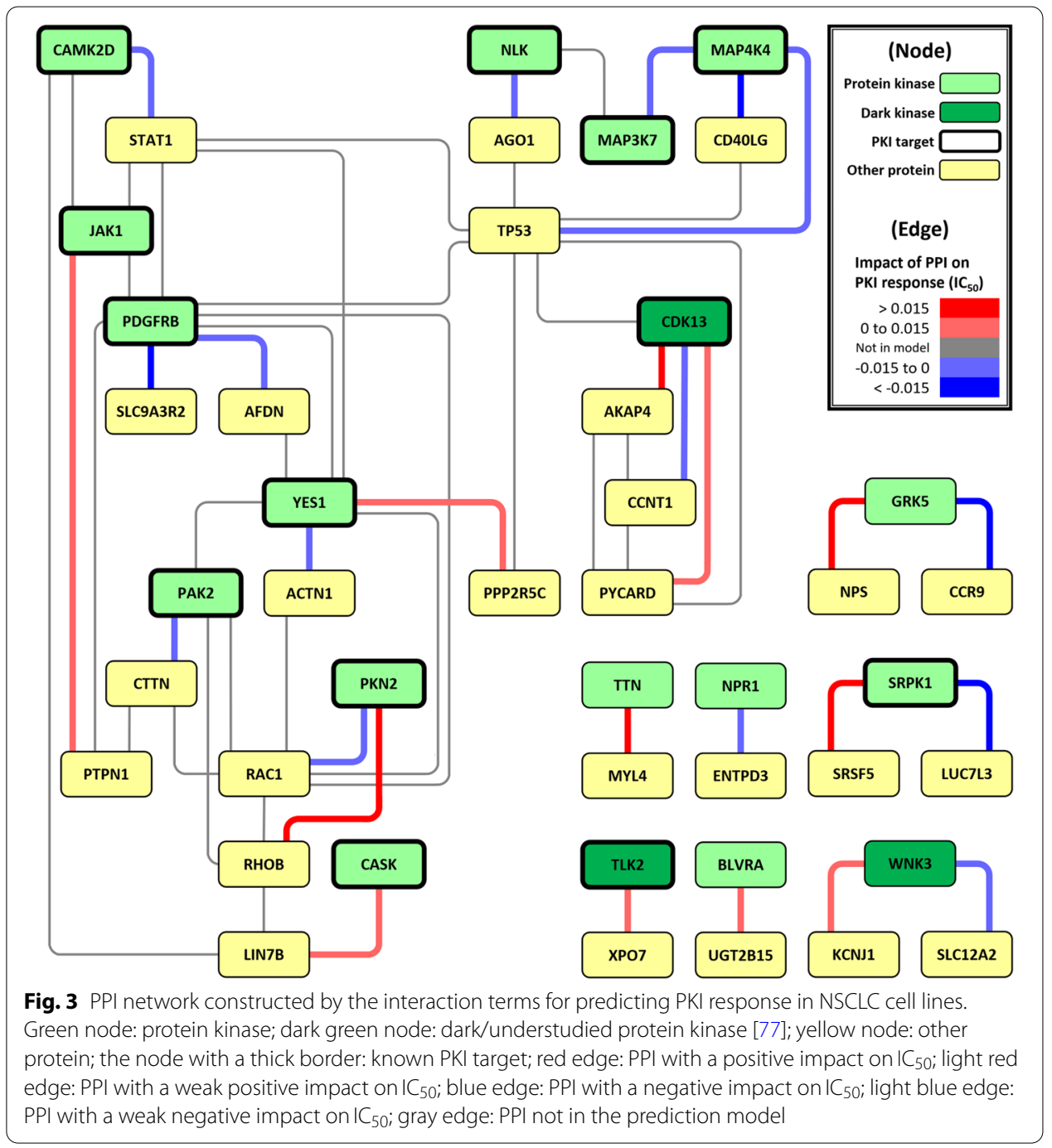

adenocarcinoma [41]. MAP4K4 expression is up-regulated upon binding to p53, resulting in the activation of the apoptotic JNK signaling pathway [42]. In the NSCLC dataset, when the expression of MAP4K4-TP53 interaction (“EXP_MAP4K4_X_EXP_TP53”) increases, the average $\mathrm{IC}_{50}$ is slightly decreased (Pearson correlation $=-0.10$ ). In the pre-trained PKI response prediction model, every unit of gene expression level increase in MAP4K4-TP53 PPI is associated with a 0.012 unit decrease in $\mathrm{IC}_{50}$ on average (average predicted $\mathrm{IC}_{50}=2.727$ versus 2.715 ), suggesting that this up-regulated PPI in apoptotic JNK signaling pathway contributes causatively to the observed drug sensitivity.

\section{Role of drug-mutation association in drug response prediction}

Finally, we wanted to investigate the extent to which drug-mutation interactions quantitatively contribute to PKI response prediction in NSCLC. In total, there are 47 drugmutation interaction terms in the NSCLC model, and they are located at 22 structural locations represented by spheres in Fig. 4a (PDB ID: 1ATP). Their impacts on $\mathrm{IC}_{50}$ are listed in Additional file 1: Table S6, sorted by absolute $\mathrm{IC}_{50}$ impact. The drug-mutation 


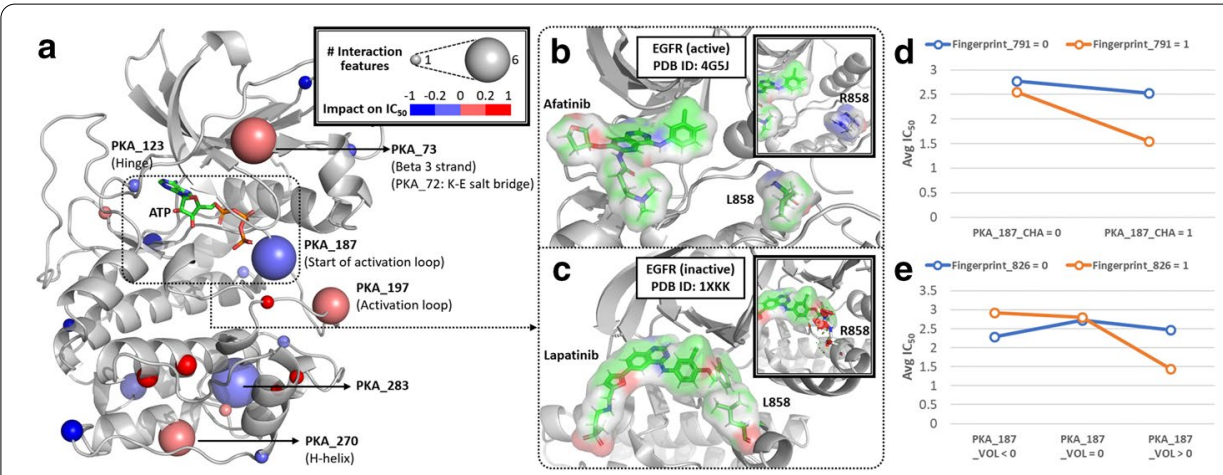

Fig. 4 Distribution of drug-mutation relationships on the reference protein kinase A (PKA) crystal structure and interaction analyses. $\mathbf{a}$ Interaction hot spots are labeled and represented by larger spheres on the reference PKA structure (PDB ID: 1ATP). If a residue is involved in multiple drug-mutation relationships, the median of their impacts on $\mathrm{IC}_{50}$ is chosen to represent the color of the sphere. Red sphere represents drugmutation relationship with a positive impact on $\mathrm{IC}_{50}$; blue sphere represents relationship with a negative impact on $\mathrm{IC}_{50}$. b, c represent examples of two PKIs (afatinib and lapatinib) with different binding modes in the active (PDB ID: 4G5J) and inactive (PDB ID: 1XKK) conformations of EGFR, respectively. The residue corresponding to EGFR L858 (PKA_187) is labeled in each example; the mutant form (arginine) modeled in PyMol [78] is shown. d, e represent statistical interaction analyses for Fingerprint_791 versus PKA_187_CHA and Fingerprint_826 versus PKA_187_VOL in the NSCLC dataset, respectively

relationships located in the canonical ATP-binding pocket (highlighted by a dashed rectangle in Fig. 4a) could be formed by type I or type II protein kinase inhibitors that bind to active or inactive kinase conformations, respectively [43]. For example, mutation mapping to the beginning of the activation segment (residue position 187 in protein kinase A ("PKA_187") is located in this pocket. In the NSCLC dataset, there are three mutations located in PKA_187: EGFR L858R, BRAF L597V, and STK32C I237V.

Figure $4 \mathrm{~b}$, c respectively show different binding modes of two EGFR inhibitors (afatinib and lapatinib) that contribute to variable response in L858R mutant EGFR. H3255, an NSCLC cell line with EGFR L858R mutation, is hypersensitive to afatinib $\left(\mathrm{IC}_{50}=-4.35\right.$; average $\mathrm{IC}_{50}=2.03$ for all the NSCLC cell lines treated with afatinib). Notably, the L858R mutation can be accommodated in the active conformation of EGFR, but not in the inactive state due to steric hindrance [44].

An interaction analysis (Fig. 4d) shows that the mutated residues involving charge difference at PKA_187 have significant interaction ( $p$ value $=0.043$, F-test $)$ with Fingerprint_791, a drug substructure "NC1CCC(N)CC1" of afatinib. Based on our prediction model, every unit increase in "PKA_187_CHA_X_Fingerprint_791", an interaction term with one of the highest impact on $\mathrm{IC}_{50}$ among all the drug-mutation interaction terms in the model (Additional file 1: Table S6), is associated with a 0.46 unit decrease in $\mathrm{IC}_{50}$ on average (average predicted $\mathrm{IC}_{50}=2.73$ versus 2.27 ). Another interaction analysis (Fig. 4e) shows that the mutated residues involving volume difference at PKA_187 have significant interaction ( $p$ value $=0.035$, F-test $)$ with Fingerprint_826, a drug substructure "OC1C $(\mathrm{N}) \mathrm{CCCC} 1$ " of afatinib. Every unit increase in "PKA_187_VOL_X_Fingerprint_826" is associated with a 0.01 unit decrease in $\mathrm{IC}_{50}$ on average (average predicted $\mathrm{IC}_{50}=2.73$ versus 2.72 ). Since lapatinib lacks both substructures Fingerprint_791 and Fingerprint_826, we speculate that mutant EGFR in NSCLC cells with a larger, positively charged mutation at PKA_187 are resistant to lapatinib (the blue lines in Fig. 4d, e). 


\section{Discussion}

In this study, we propose a PKI response prediction framework to estimate $\mathrm{IC}_{50}$ values with a more explainable AI model. This framework includes four components: (1) drug features and cancer cell line's multi-omics features, (2) statistical tests for capturing interaction effects, (3) feature selection, and (4) machine learning methods. We validated the contribution of each component and used the NSCLC dataset as a case study to explain the contributing features in PKI response prediction.

The intrinsic limitation of drug response prediction is the unexplainable variation of drug response caused by different assays and experimental conditions. Several previous studies on drug response prediction used data not only from GDSC but also from CCLE (Table 1). However, Juan-Blanco et al. [26] pointed out that although GDSC and CCLE datasets shared 343 cancer cell lines and 15 drugs, the drug responses from these two datasets were poorly correlated. Thus, we only used a single source in this study to minimize the unexplainable effect from different experimental conditions. Nevertheless, this situation impeded us from finding appropriate independent testing set outside the GDSC data. Even though the drug response data we used were only from GDSC, the feature selection process showed that the drug feature "From_Sanger" was selected for all the 23 cancer-centric prediction models, meaning that the batch effects are significant depending on the origin of datasets (Wellcome Sanger Institute vs. Massachusetts General Hospital). The GDSC 8.0 dataset was released while our studies were underway. Compared with release 7.0, it contains 160 thousand more drug responses. However, this dramatic increase does not provide us with an appropriate test set, because the old drug response dataset (called GDSC1 in release 8.0) and the new drug response dataset (called GDSC2) were generated based on different experimental protocols. Furthermore, PKI responses in the two datasets show a weak correlation $\left(R^{2}=0.6\right.$, Additional file 1 : Figure S2).

Our study has revealed different interaction terms and types contributing to the prediction of drug response profiles in cell-based assays. The QSMART model can potentially be extended to other applications, such as protein-ligand interaction, gene-environment interaction, and agent-host interaction. However, in addition to the unexplainable variation issue mentioned above, improving generalization performance is challenging for prediction models with multiple interaction terms, which require more samples to detect significant interactions [45]. We randomly removed $10 \%$ of the samples and compared the selected features of these reduced training sets with those of full training sets. We found that the full training sets' 1896 (81.4\%) features, including $75.4 \%$ of the interaction terms, were still selected in the reduced sets (Additional file 1: Table S7). The features discussed in the Case study, "From_Sanger”, “GO_0030324_X_GO_0048675", “EXP_MAP4K4_X_EXP_TP53", and "PKA_187_VOL_X_Fingerprint_826" were still selected in the reduced NSCLC set. Although "PKA_187_CHA_X_Fingerprint_791" was not selected, a relevant interaction term about the polarity change "PKA_187_ POL_X_Fingerprint_791" was in the reduced NSCLC set. Nevertheless, 164 interaction terms were uniquely selected in the reduced sets. These unique interaction terms also showed statistical significance to drug response prediction in the full sets, but the feature selection methods did not select them under the BIC control. Although the number of training samples was reduced, more than three-quarters of the features were still in 
the models, and the overall performance did not significantly change (Additional file 1: Table S8). To increase generalization performance and the stability of our prediction framework, increasing the sample size will help. Thus, when people apply the concept of QSMART to other interaction types, sample size and sample availability should be considered.

\section{Conclusions}

In conclusion, by integrating multi-omics data in the QSMART model, we not only predict PKI responses in cancer cell lines with high accuracy but also identify features and interaction terms contributing to the accuracy, thereby enhancing the explainability of the prediction models. Compared to traditional QSAR models, the QSMART model proposed in this study further introduces different types of interaction terms, which are usually hidden in deep neural network models. While we demonstrate our model in protein kinase inhibitor binding, the QSMART model can be applied to other druggable gene families such as $\mathrm{G}$ protein-coupled receptors (GPCRs).

\section{Methods}

\section{Framework for drug response prediction}

The overall objective of this study is to emphasize the contribution of interaction terms that capture drug-mutation relationships and to show how these interaction terms could help explain the mechanism of drug response. The framework we propose in this study includes four main components: (1) the substructure fingerprints of protein kinase inhibitor (PKI) and cancer cell line's multi-omics features, including from low-level features, such as residue mutations, to high-level features, such as perturbed biological processes, (2) F-test for identifying significant drug-mutation relationships and other interaction effects, (3) a feature selection method: Lasso with Bayesian information criterion (BIC) control, and (4) a machine learning method to predict PKI response: neural networks (Fig. 1). The modular nature of this framework provides flexibility by allowing each component to be updated independently based on new datasets and methodology. To implement this framework, we collected a dataset containing 0.2 million drug responses $\left(\mathrm{IC}_{50}\right.$ in a logarithmic scale; "IC 50 " hereinafter) from GDSC, split them into 23 sub-datasets according to the primary site where the cancer cell line originated, and then built a cancer-centric model for each sub-dataset. More details about each component are described below.

\section{Protein kinase inhibitor}

We define small-molecule (molecular weight $<900$ daltons) protein kinase inhibitors in GDSC from a variety of publicly available, manually curated drug-target databases, and experimental data. The list of human protein kinases in this study is defined by ProKinO [46] (version 2.0). Drug-kinase associations were extracted from DrugBank [47] (version 5.1.0), Therapeutic Target Database (TTD [48], last accessed on September 15th, 2017), Pharos [49] (last accessed on May 15th, 2018), and LINCS Data Portal [50] (last accessed on May 15th, 2018). We define a drug as a PKI if it is annotated as an "inhibitor", "antagonist", or "suppressor" in the drug-kinase associations. We also include the PKIs in LINCS Data Portal if their controls are less than 5\% in KINOMEscan assays. Based on 
these criteria, we define 143 small-molecule PKIs out of the 252 unique screened compounds in GDSC (Additional file 4).

\section{Drug response}

GDSC (release 7.0) provides the half-maximal inhibitory concentration values $\left(\mathrm{IC}_{50}\right)$ for 224,202 drug-cancer cell line pairs. The drug sensitivity assays were performed either by the Wellcome Trust Sanger Institute or the Massachusetts General Hospital Cancer Center. In this drug response dataset, there are 12,509 duplicate drug-cancer cell line pairs due to 16 duplicate drugs. We measured the Pearson correlation coefficient between the $\mathrm{IC}_{50}$ values of each duplicate drug in the two assays. Only afatinib and refametinib showed a strong positive correlation $(\mathrm{r}>0.7)$; their $\mathrm{IC}_{50}$ values were then merged by their weighted means [51]. We exclude duplicate drugs with a correlation coefficient of less than 0.7 from our study. The resulting dataset of 197,459 nonredundant drug responses consists of 236 drugs and 1065 cancer cell lines. After filtering out non-PKIs, 109,856 non-redundant drug responses consisting of 135 PKIs and 1064 cancer cell lines remained.

\section{Drug features}

The 2D structures of drugs were obtained from PubChem in SDF format. The Chemistry Development Kit Descriptor Calculator Graphical User Interface [52] (version 1.4.6) generated 881 PubChem fingerprints and 286 chemical descriptors, including constitutional, topological, electronic, geometric, and bridge descriptors. Observing high redundancy and multicollinearity within features, we removed redundant features and implemented the variance inflation factor criterion (VIF) [53] to reduce multicollinearity (for more details, see the Feature screening section). After filtering, 92 PubChem fingerprints and 0 chemical descriptors remained.

\section{Cancer cell line features}

Using mutation profiles for each cancer cell line sample provided by COSMIC Cell Lines Project [54] (v87), we incorporate 7 categories of multi-omics features to quantify the differences between wild type and mutant protein kinases:

1 Residue-level: reference protein kinase A (PKA) position (from ProKinO), mutant type, charge, polarity, hydrophobicity, accessible surface area, side-chain volume, energy per residue [55], and substitution score (BLOSUM62 [56])

2 Motif-level: sequence and structural motifs of protein kinase (from ProKinO)

3 Domain-level: subdomain in protein kinase (from ProKinO) and functional domain (from Pfam [57] v31.0)

4. Gene-level: the number of mutations in the genes encoding protein kinases, gene expression (from GDSC), and copy number variation (from COSMIC)

5 Family-level: protein kinase family and group (from ProKinO)

6 Pathway-level: reaction, pathway (from Reactome [58], last accessed on May 15th, 2018), and biological process (from AmiGO [59], last accessed on May 15th, 2018)

7 Sample-level: microsatellite instability, average ploidy, age, cancer originated tissue type, and histological classification (from COSMIC and Cellosaurus, [60]). 
The formulas for generating all cancer cell line features are shown in Additional file 1: Table S9.

\section{QSMART model}

The Quantitative Structure-Mutation-Activity Relationship Tests (QSMART) model was developed based on the QSAR model. First, we built a basic model with all drug features and cancer cell line features as independent variables for estimating $\mathrm{IC}_{50}$ :

$$
I C_{50}=\beta_{0}+\sum_{i=1}^{I} \beta_{1 i} D_{i}+\sum_{j=1}^{J} \beta_{2 j} C_{j}+\epsilon,
$$

where $\beta_{0}$ is the intercept, $\beta_{1 i}$ and $\beta_{2 j}$ are the coefficients of the $i$ th drug feature $D_{i}$ and the $j$ th cancer cell line feature $C_{j}$, and $\epsilon$ is the error term.

Because the residue-level features of a cancer cell line represent the mutation status in the reference PKA structure, and we are interested in investigating drug-mutation relationships, we introduced drug-mutation interaction terms in the model:

$$
I C_{50}=\beta_{0}+\sum_{i=1}^{I} \beta_{1 i} D_{i}+\sum_{j=1}^{J} \beta_{2 j} C_{j}+\sum_{i=1}^{I} \sum_{k=1}^{K} \beta_{3 i k} D_{i} M_{k}+\epsilon,
$$

where $\beta_{3 i k}$ is the coefficient of the interaction term formed by the $i$ th drug feature $D_{i}$ and the $k$ th residue-level feature $M_{k}$. Since all cancer cell line features contain residue-level features and the other six feature categories, $\left\{C_{1}, \ldots, C_{J}\right\}$ is a superset of $\left\{M_{1}, \ldots, M_{K}\right\}$. Considering that the interaction terms formed by the substructures of drug and highlevel cancer cell line features have no biological relevance, we did not incorporate all cancer cell line features as part of interaction terms. For example, we did not consider the interaction between a substructure "Fingerprint_1" and a biological process "lung development" because it is unexplainable.

In addition to using all cancer cell line features, we further introduced additional interaction terms to capture various proteomic, cellular, and genomic features:

$$
\begin{aligned}
I C_{50}= & \beta_{0}+\sum_{i=1}^{I} \beta_{1 i} D_{i}+\sum_{j=1}^{J} \beta_{2 j} C_{j}+\sum_{i=1}^{I} \sum_{k=1}^{K} \beta_{3 i k} D_{i} M_{k} \\
& +\sum_{p=1}^{P} \beta_{4 p} P P I_{p}+\sum_{q=1}^{Q} \beta_{5 q} R E C x_{q}+\sum_{r=1}^{R} \beta_{6 r} P W Y x_{r}+\sum_{s=1}^{S} \beta_{7 s} G O x_{s}+\epsilon,
\end{aligned}
$$

where $\beta_{4 p}, \beta_{5 q}, \beta_{6 r}$, and $\beta_{7 s}$ are the coefficients of the $p$ th protein-protein interaction $P P I_{p}$, the $q$ th reaction-reaction interaction $R E C x_{q}$, the $r$ th pathway-pathway interaction $P W Y x_{r}$, and the $s$ th biological process interaction GOx , respectively. More details about interaction terms are described below.

\section{Interaction terms}

Five types of interaction terms were introduced into the QSMART model: drug-mutation interaction, protein-protein interaction, reaction-reaction interaction, pathway-pathway interaction, and biological process interaction. These interactions were 
not necessarily physical; instead, they were predictors that show statistically significant contribution to explaining the variation of $\mathrm{IC}_{50}$ values. For drug-mutation interaction terms, only the residue mapping to the reference PKA structure was considered to form interactions with drugs. For protein-protein interaction (PPI), we retained the non-selfinteraction PPIs formed by at least one human protein kinase with interaction scores greater than 700 in the STRING database [61]. Gene expression level was used as a weight for PPIs to represent protein levels in cancer cell lines. For reaction, pathway, and biological process interactions, we removed the interactions formed by two entities from the same biological process/pathway hierarchy. For instance, the interaction between the biological process "lung cell differentiation" (GO:0060479) and its parent "lung development" (GO:0030324) was removed since it is unexplainable. Each interaction term was tested individually by F-test using R [62] (version 3.4.4). Significant interaction terms $(\mathrm{FDR}<0.05)$ with no less than 30 non-zero values were used for further feature selection.

\section{Datasets}

To reduce potential sources of noise and bias, we further filtered cancer cell lines from the PKI response dataset if (1) their mutation profiles are not detected by whole-genome sequencing, (2) they have less than 30 drug response entries, (3) their gene expression profile is not available, or (4) their mutation site does not map to a residue in the reference PKA position. The dataset was then split into 29 groups, stratified by primary cancer sites. Groups with less than 1000 responses (adrenal gland, biliary tract, placenta, prostate, salivary gland, small intestine, testis, and vulva) were excluded due to low statistical power. "Haematopoietic and lymphoid tissue", the largest group, was further divided into two subsets by primary histology: "haematopoietic neoplasm" and "lymphoid neoplasm". For the case study, we collected cancer cell lines for the non-small cell lung cancer (NSCLC) dataset from the lung cancer dataset if their histology subtype is adenocarcinoma, non-small cell carcinoma, squamous cell carcinoma, large cell carcinoma, giant cell carcinoma, or mixed adenosquamous carcinoma. Remaining lung cancer cell samples were classified as "lung (others)". We created cancer type-centric training sets by expanding the drug response dataset with drug features, cancer cell lines features, and significant interaction terms. Categorical data in the training sets were coded into dummy variables. As a result, we prepared 23 cancer type-centric training sets. The number of PKI responses for each cancer type is shown in Table 2.

\section{Feature screening}

Observing high multicollinearity within the features in the first component of our prediction framework (Fig. 1), we implemented the variance inflation factor criterion (VIF) [53] to remove highly correlated features. For the multiple regression model with $f$ features, $X_{i}(i=1, \ldots, f)$, the VIF for the $i$ th feature can be expressed by: $V I F_{i}=\frac{1}{1-R_{i}^{2}}$, where $R_{i}^{2}$ is the coefficient of determination of the regression between $X_{i}$ and the remaining $f-1$ features. $V I F_{i}>5$ (i.e. $R_{i}^{2}>0.8$ ) is considered to be high collinearity and $X_{i}$ should be excluded from the model [53]. We first prioritized drug features based on these rules: (1) the later PubChem fingerprint bit positions (complex patterns) have higher priorities than the earlier ones (simple elements), and (2) PubChem fingerprints 
have higher priorities than calculated chemical descriptors because fingerprints directly represent molecular substructures of the drug. Chemical descriptors, such as ALogP [63], are calculated or estimated based on multiple substructures. In our study, because we considered the interactions between these high-level drug features and mutations were not easily explainable, we chose to assign low priorities to these drug features when performing feature screening. This process can be viewed as feature engineering based on domain knowledge [64]. Essentially, if experts understand what the features mean, they will better interpret the model. Then, we implemented stepwise selection (starting from higher priority features) under VIF control. Co-expressed genes in the same prediction model also exhibited collinearity. To address this issue, we also used the VIF criterion to filter co-expressed genes in each training set.

\section{Feature selection}

To combat the problem of $\mathrm{p}$ (the number of drug features plus cancer cell line features and interaction terms) $>>\mathrm{n}$ (the number of drug responses) in the training sets, we implemented Lasso [65] with Bayesian Information Criterion (BIC) [66] by an R package "HDeconometrics" [67]. Lasso is appropriate for estimating coefficients in high-dimensional space [68], while BIC provides an efficient approach to select the optimal Lasso model [69]. Under the condition of a fixed number of drug responses, the model was penalized based on the number of selected features when minimizing BIC:

$$
B I C=k \cdot \ln (n)-2 \ln (\widehat{L}),
$$

where $\widehat{L}$ is the maximum likelihood of the model, $\mathrm{k}$ is the number of features in the model, and $\mathrm{n}$ is the number of observations (drug responses) used in the model. After feature selection, the remaining number of selected features for each cancer type is shown in Table 2.

Additionally, we performed three distinct feature selection methods with different underlying assumptions and one ensemble method. We used WEKA's correlation attribute evaluation, ReliefF, and classifier (random forests) attribute evaluation to rank features [70], and then calculated each feature's average rank in Lasso and these three methods. To make the results comparable, we selected the same number of features as those we selected using Lasso under BIC control.

\section{Neural network architecture}

We built neural network models by using JMP ${ }^{\circ}$ [71]. We designed three types of neural network architectures in this study: single-layer, double-layer, and complex-double-layer. The numbers of hidden layer nodes follow the geometric pyramid rule [72]. Given N input nodes objectively determined by the feature selection methods, there are $\left\lceil N^{1 / 2}\right\rceil$ hidden nodes in a single-layer architecture. In a double-layer architecture, there are $\left\lceil N^{2 / 3}\right\rceil$ and $\left\lceil N^{1 / 3}\right\rceil$ hidden nodes in the first and second hidden layers, respectively. In a complex-double-layer architecture, there are $\mathrm{N}$ and $\left\lceil N^{1 / 2}\right\rceil$ hidden nodes in the first and second hidden layers, respectively. The nodes among the two layers are fully connected. Biases are introduced into the input and hidden layers. The activation function of every node is a hyperbolic tangent function (TanH). A quasi-Newton method, BFGS [73], is chosen as an optimizer by JMP. 
To mitigate overfitting, we performed 10 -fold cross-validation, early stopping, and Lasso-style penalty function (absolute value penalty, i.e., $\mathrm{L}_{1}$ regularization [74]). When performing 10-fold cross-validation, we partitioned the observations (drug responses) into ten folds. In turn, each fold served as a validation set to evaluate the model built upon the rest nine folds. The tuning parameters that construct the model giving the best validation statistics were selected in the final model. The average performance $\left(R^{2}\right)$ of the ten models for each cancer type was reported. To tune the hyperparameters, we started from a single-layer model for each cancer type based on Occam's razor principle [75]. If the performance is less than the threshold of 0.8 in 200 iterations, we increased the number of iterations to 300; if the performance is still less than the threshold, we implemented a double-layer model for 200 iterations, and so on until using a complex-doublelayer model for 300 iterations.

\section{Other machine learning and drug responses prediction methods}

We compared neural networks with three other prediction algorithms with 10-fold cross-validation: random forests, support vector machine (SVM), and elastic net. Random forests were implemented by WEKA [70] (version 3.8.3). For each cancer type, the number of iterations was decided based on the iterations used for each of the pre-trained neural network models (200 or 300 iterations) shown in Additional file 1: Table S1. SVM was implemented by the SMOreg function (SVM for regression) of WEKA. Elastic net was implemented by an R package "glmnet" [76]. To optimize the parameter settings for the compared machine learning methods, we used the grid search method. We built 100 models with different parameter combinations for each method. Detailed parameter values are available in Additional file 5.

Additionally, we also compared our prediction models with two-way ANOVA analysis and a drug response prediction model, multiscale convolutional attentive (MCA) [36]. Because the purpose of two-way ANOVA analysis implemented by $\mathrm{R}$ was to quantify how much two factors (drug and cancer cell line) can explain the variation of drug response (adjusted $R^{2}$ was used), the model used the drug and cancer cell line identifiers as inputs and did not undergo 10-fold cross-validation. MCA combines gene expression profiles, the molecular structure of compounds, and prior knowledge of protein-protein interactions, and uses convolutional neural networks to predict drug response. The performance of MCA for PKI response prediction is available in Additional file 2.

\section{Supplementary information}

Supplementary information accompanies this paper at https://doi.org/10.1186/s12859-020-03842-6.

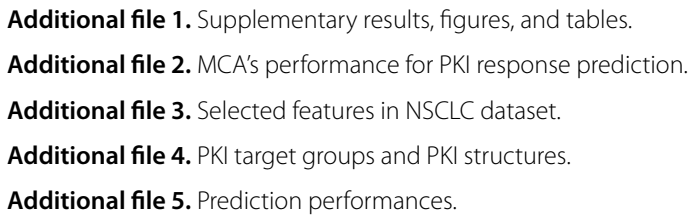


relationship; QSMART: Quantitative structure-mutation-activity relationship tests; SVM: Support vector machine; VIF: Variance inflation factor; XAl: Explainable artificial intelligence.

\section{Acknowledgements}

Not applicable.

\section{Authors' contributions}

LH, PM, KR, and NK designed the research. LH performed data integration. LH, YW, HC, and PM performed statistical analyses. $L H, Y W, H C$, SL, and KR performed machine learning methods. $L H, W Y, Y W, H C, P M$, and NK analyzed the data and interpreted the results. LH, WY, AV, and NK wrote the manuscript. LH created the tables and figures. YW, HC, SL, PM, and KR revised the manuscript. All authors read and approved the final manuscript.

\section{Funding}

This work has been supported by National Institutes of Health (funding for NK from U01CA239106; funding for PM from R01GM122080) and National Science Foundation (funding for PM from DMS-1903226). PM, YW, and HC were partially supported by NIH grants R01 GM113242, R01 GM122080, NSF grants DMS-1925066 and DMS-1903226. The funding bodies did not play any roles in the design of the study and collection, analysis, and interpretation of data and in writing the manuscript.

\section{Availability of data and materials}

Training sets, the codes for building prediction models, and prediction results are available at https://github.com/esbgk annan/QSMART/.

\section{Ethics approval and consent to participate}

Not applicable.

\section{Consent for publication}

Not applicable.

\section{Competing interests}

The authors declare that they have no competing interests.

\section{Author details}

${ }^{1}$ Institute of Bioinformatics, University of Georgia, 120 Green St., Athens, GA 30602, USA. ${ }^{2}$ Department of Statistics, University of Georgia, 310 Herty Drive, Athens, GA 30602, USA. ${ }^{3}$ Department of Biochemistry and Molecular Biology, 120 Green St., Athens, GA 30602, USA. ${ }^{4}$ Department of Computer Science, 415 Boyd Graduate Studies Research Center, Athens, GA 30602, USA.

Received: 9 May 2020 Accepted: 27 October 2020

Published online: 12 November 2020

\section{References}

1. Lehne G, Elonen E, Baekelandt M, Skovsgaard T, Peterson C. Challenging drug resistance in cancer therapy-review of the First Nordic Conference on Chemoresistance in Cancer Treatment, October 9th and 10th, 1997. Acta Oncol. 1998;37(5):431-9.

2. Holohan C, Van Schaeybroeck S, Longley DB, Johnston PG. Cancer drug resistance: an evolving paradigm. Nat Rev Cancer. 2013;13(10):714-26.

3. Sharma SV, Bell DW, Settleman J, Haber DA. Epidermal growth factor receptor mutations in lung cancer. Nat Rev Cancer. 2007;7(3):169-81.

4. Arslan MA, Kutuk O, Basaga H. Protein kinases as drug targets in cancer. Curr Cancer Drug Targets. 2006;6(7):623-34.

5. Bell DW, Gore I, Okimoto RA, Godin-Heymann N, Sordella R, Mulloy R, Sharma SV, Brannigan BW, Mohapatra G, Settleman J, Haber DA. Inherited susceptibility to lung cancer may be associated with the T790M drug resistance mutation in EGFR. Nat Genet. 2005;37(12):1315-6.

6. Tracy S, Mukohara T, Hansen M, Meyerson M, Johnson BE, Janne PA. Gefitinib induces apoptosis in the EGFRL858R non-small-cell lung cancer cell line H3255. Cancer Res. 2004;64(20):7241-4.

7. Pao W, Miller VA, Politi KA, Riely GJ, Somwar R, Zakowski MF, Kris MG, Varmus H. Acquired resistance of lung adenocarcinomas to gefitinib or erlotinib is associated with a second mutation in the EGFR kinase domain. PLoS Med. 2005;2(3):73.

8. Yang W, Soares J, Greninger P, Edelman EJ, Lightfoot H, Forbes S, Bindal N, Beare D, Smith JA, Thompson IR, Ramaswamy S, Futreal PA, Haber DA, Stratton MR, Benes C, McDermott U, Garnett MJ. Genomics of Drug Sensitivity in Cancer (GDSC): a resource for therapeutic biomarker discovery in cancer cells. Nucleic Acids Res. 2013;41(Database issue):955-61.

9. Barretina J, Caponigro G, Stransky N, Venkatesan K, Margolin AA, Kim S, Wilson CJ, Lehar J, Kryukov GV, Sonkin D, Reddy A, Liu M, Murray L, Berger MF, Monahan JE, Morais P, Meltzer J, Korejwa A, Jane-Valbuena J, Mapa FA, Thibault J, Bric-Furlong E, Raman P, Shipway A, Engels IH, Cheng J, Yu GK, Yu J, Aspesi P, de Silva M, Jagtap K, Jones MD, Wang L, Hatton C, Palescandolo E, Gupta S, Mahan S, Sougnez C, Onofrio RC, Liefeld T, MacConaill L, Winckler W, Reich M, Li N, Mesirov JP, Gabriel SB, Getz G, Ardlie K, Chan V, Myer VE, Weber BL, Porter J, Warmuth M, Finan P, Harris JL, Meyerson M, Golub TR, Morrissey MP, Sellers WR, Schlegel R, Garraway LA. The Cancer Cell Line Encyclopedia enables predictive modelling of anticancer drug sensitivity. Nature. 2012;483(7391):603-7. 
10. Menden MP, lorio F, Garnett M, McDermott U, Benes CH, Ballester PJ, Saez-Rodriguez J. Machine learning prediction of cancer cell sensitivity to drugs based on genomic and chemical properties. PLoS One. 2013;8(4):61318.

11. Jang IS, Neto EC, Guinney J, Friend SH, Margolin AA. Systematic assessment of analytical methods for drug sensitivity prediction from cancer cell line data. Pac Symp Biocomput. 2014;2014:63-74.

12. Geeleher P, Cox NJ, Huang RS. Clinical drug response can be predicted using baseline gene expression levels and in vitro drug sensitivity in cell lines. Genome Biol. 2014;15(3):47.

13. Dong Z, Zhang N, Li C, Wang H, Fang Y, Wang J, Zheng X. Anticancer drug sensitivity prediction in cell lines from baseline gene expression through recursive feature selection. BMC Cancer. 2015;15:489.

14. Zhang N, Wang H, Fang Y, Wang J, Zheng X, Liu XS. Predicting anticancer drug responses using a dual-layer integrated cell line-drug network model. PLoS Comput Biol. 2015;11(9):1004498.

15. Gupta S, Chaudhary K, Kumar R, Gautam A, Nanda JS, Dhanda SK, Brahmachari SK, Raghava GP. Prioritization of anticancer drugs against a cancer using genomic features of cancer cells: a step towards personalized medicine. Sci Rep. 2016;6:23857.

16. Ammad-Ud-Din M, Khan SA, Malani D, Murumagi A, Kallioniemi O, Aittokallio T, Kaski S. Drug response prediction by inferring pathway-response associations with kernelized Bayesian matrix factorization. Bioinformatics. 2016;32(17):455-63.

17. Nguyen L, Dang CC, Ballester PJ. Systematic assessment of multi-gene predictors of pan-cancer cell line sensitivity to drugs exploiting gene expression data. F1000Res. 2017;5:2927.

18. Stanfield Z, Coskun M, Koyuturk M. Drug response prediction as a link prediction problem. Sci Rep. 2017;7:40321.

19. Ammad-Ud-Din M, Khan SA, Wennerberg K, Aittokallio T. Systematic identification of feature combinations for predicting drug response with Bayesian multi-view multi-task linear regression. Bioinformatics. 2017;33(14):359-68.

20. Geeleher P, Zhang Z, Wang F, Gruener RF, Nath A, Morrison G, Bhutra S, Grossman RL, Huang RS. Discovering novel pharmacogenomic biomarkers by imputing drug response in cancer patients from large genomics studies. Genome Res. 2017:27(10):1743-51.

21. Rahman R, Matlock K, Ghosh S, Pal R. Heterogeneity aware random forest for drug sensitivity prediction. Sci Rep. 2017;7(1):11347.

22. Ding MQ, Chen L, Cooper GF, Young JD, Lu X. Precision oncology beyond targeted therapy: combining omics data with machine learning matches the majority of cancer cells to effective therapeutics. Mol Cancer Res. 2018;16(2):269-78.

23. Chang Y, Park H, Yang HJ, Lee S, Lee KY, Kim TS, Jung J, Shin JM. Cancer Drug Response Profile scan (CDRscan): a deep learning model that predicts drug effectiveness from cancer genomic signature. Sci Rep. 2018;8(1):8857.

24. Cichonska A, Pahikkala T, Szedmak S, Julkunen H, Airola A, Heinonen M, Aittokallio T, Rousu J. Learning with multiple pairwise kernels for drug bioactivity prediction. Bioinformatics. 2018;34(13):509-18.

25. He X, Folkman L, Borgwardt K. Kernelized rank learning for personalized drug recommendation. Bioinformatics. 2018;34(16):2808-16.

26. Juan-Blanco T, Duran-Frigola M, Aloy P. Rationalizing drug response in cancer cell lines. J. Mol. Biol. 2018;430(18 Pt A):3016-27.

27. Le DH, Pham VH. Drug response prediction by globally capturing drug and cell line information in a heterogeneous network. J Mol Biol. 2018;430(18 Pt A):2993-3004.

28. Liu H, Zhao Y, Zhang L, Chen X. Anti-cancer Drug Response Prediction Using Neighbor-Based Collaborative Filtering with Global Effect Removal. Mol Ther Nucleic Acids. 2018;13:303-11.

29. Wei D, Liu C, Zheng X, Li Y. Comprehensive anticancer drug response prediction based on a simple cell line-drug complex network model. BMC Bioinformatics. 2019;20(1):44.

30. Wang X, Sun Z, Zimmermann MT, Bugrim A, Kocher JP. Predict drug sensitivity of cancer cells with pathway activity inference. BMC Med Genomics. 2019;12(Suppl 1):15.

31. Chiu YC, Chen HH, Zhang T, Zhang $S$, Gorthi A, Wang LJ, Huang Y, Chen Y. Predicting drug response of tumors from integrated genomic profiles by deep neural networks. BMC Med Genomics. 2019;12(Suppl 1):18.

32. Li Q, Shi R, Liang F. Drug sensitivity prediction with high-dimensional mixture regression. PLoS One. 2019;14(2):0212108.

33. Yang J, Li A, Li Y, Guo X, Wang M. A novel approach for drug response prediction in cancer cell lines via network representation learning. Bioinformatics. 2019;35(9):1527-35.

34. Lind AP, Anderson PC. Predicting drug activity against cancer cells by random forest models based on minimal genomic information and chemical properties. PLoS ONE. 2019;14(7):0219774.

35. Liu P, Li H, Li S, Leung KS. Improving prediction of phenotypic drug response on cancer cell lines using deep convolutional network. BMC Bioinformatics. 2019;20(1):408.

36. Manica M, Oskooei A, Born J, Subramanian V, Saez-Rodriguez J, Rodriguez Martinez M. Toward explainable anticancer compound sensitivity prediction via multimodal attention-based convolutional encoders. Mol Pharm. 2019;16(12):4797-806.

37. Oskooei A, Manica M, Mathis R, Martinez MR. Network-based Biased Tree Ensembles (NetBiTE) for drug sensitivity prediction and drug sensitivity biomarker identification in cancer. Sci Rep. 2019;9(1):15918.

38. Gunning D, Aha DW. Darpa's explainable artificial intelligence program. Al Mag. 2019;40(2):44-58.

39. Chedotal A, Kerjan G, Moreau-Fauvarque $C$. The brain within the tumor: new roles for axon guidance molecules in cancers. Cell Death Differ. 2005;12(8):1044-56.

40. Thomas PD, Campbell MJ, Kejariwal A, Mi H, Karlak B, Daverman R, Diemer K, Muruganujan A, Narechania A. PANTHER: a library of protein families and subfamilies indexed by function. Genome Res. 2003;13(9):2129-41.

41. Qiu MH, Qian YM, Zhao XL, Wang SM, Feng XJ, Chen XF, Zhang SH. Expression and prognostic significance of MAP4K4 in lung adenocarcinoma. Pathol Res Pract. 2012;208(9):541-8.

42. Miled C, Pontoglio M, Garbay S, Yaniv M, Weitzman JB. A genomic map of p53 binding sites identifies novel p53 targets involved in an apoptotic network. Cancer Res. 2005;65(12):5096-104.

43. Gavrin LK, Saiah E. Approaches to discover non-atp site kinase inhibitors. MedChemComm. 2013;4(1):41-51. 
44. Yun CH, Boggon TJ, Li Y, Woo MS, Greulich H, Meyerson M, Eck MJ. Structures of lung cancer-derived EGFR mutants and inhibitor complexes: mechanism of activation and insights into differential inhibitor sensitivity. Cancer Cell. 2007;11(3):217-27.

45. Leon AC, Heo M. Sample sizes required to detect interactions between two binary fixed-effects in a mixedeffects linear regression model. Comput Stat Data Anal. 2009;53(3):603-8.

46. McSkimming DI, Dastgheib S, Talevich E, Narayanan A, Katiyar S, Taylor SS, Kochut K, Kannan N. ProKinO: a unified resource for mining the cancer kinome. Hum Mutat. 2015;36(2):175-86.

47. Wishart DS, Feunang YD, Guo AC, Lo EJ, Marcu A, Grant JR, Sajed T, Johnson D, Li C, Sayeeda Z, Assempour N, lynkkaran I, Liu Y, Maciejewski A, Gale N, Wilson A, Chin L, Cummings R, Le D, Pon A, Knox C, Wilson M. DrugBank 5.0: a major update to the DrugBank database for 2018. Nucleic Acids Res. 2018;46(D1):1074-82.

48. Li YH, Yu CY, Li XX, Zhang P, Tang J, Yang Q, Fu T, Zhang X, Cui X, Tu G, Zhang Y, Li S, Yang F, Sun Q, Qin C, Zeng X, Chen Z, Chen YZ, Zhu F. Therapeutic target database update 2018: enriched resource for facilitating bench-toclinic research of targeted therapeutics. Nucleic Acids Res. 2018;46(D1):1121-7.

49. Nguyen DT, Mathias S, Bologa C, Brunak S, Fernandez N, Gaulton A, Hersey A, Holmes J, Jensen LJ, Karlsson A, Liu G, Ma'ayan A, Mandava G, Mani S, Mehta S, Overington J, Patel J, Rouillard AD, Schurer S, Sheils T, Simeonov A, Sklar LA, Southall N, Ursu O, Vidovic D, Waller A, Yang J, Jadhav A, Oprea TI, Guha R. Pharos: collating protein information to shed light on the druggable genome. Nucleic Acids Res. 2017;45(D1):995-1002.

50. Koleti A, Terryn R, Stathias V, Chung C, Cooper DJ, Turner JP, Vidovic D, Forlin M, Kelley TT, D'Urso A, Allen BK, Torre D, Jagodnik KM, Wang L, Jenkins SL, Mader C, Niu W, Fazel M, Mahi N, Pilarczyk M, Clark N, Shamsaei B, Meller J, Vasiliauskas J, Reichard J, Medvedovic M, Ma'ayan A, Pillai A, Schurer SC. Data Portal for the Library of Integrated Network-based Cellular Signatures (LINCS) program: integrated access to diverse large-scale cellular perturbation response data. Nucleic Acids Res. 2018;46(D1):558-66.

51. Jones DC, Hallyburton I, Stojanovski L, Read KD, Frearson JA, Fairlamb AH. Identification of a K-opioid agonist as a potent and selective lead for drug development against human African trypanosomiasis. Biochem Pharmacol. 2010;80(10):1478-86.

52. Steinbeck C, Hoppe C, Kuhn S, Floris M, Guha R, Willighagen EL. Recent developments of the chemistry development kit (CDK) - an open-source java library for chemo- and bioinformatics. Curr Pharm Des. 2006;12(17):2111-20.

53. Akinwande MO, Dikko HG, Samson A, et al. Variance inflation factor: as a condition for the inclusion of suppressor variable (s) in regression analysis. Open J Stat. 2015;5(07):754.

54. Tate JG, Bamford S, Jubb HC, Sondka Z, Beare DM, Bindal N, Boutselakis H, Cole CG, Creatore C, Dawson E, Fish P, Harsha B, Hathaway C, Jupe SC, Kok CY, Noble K, Ponting L, Ramshaw CC, Rye CE, Speedy HE, Stefancsik R, Thompson SL, Wang S, Ward S, Campbell PJ, Forbes SA. COSMIC: the catalogue of somatic mutations in cancer. Nucleic Acids Res. 2019;47(D1):941-7.

55. Kawashima S, Ogata H, Kanehisa M. AAindex: amino acid index database. Nucleic Acids Res. 1999;27(1):368-9.

56. Henikoff S, Henikoff JG. Amino acid substitution matrices from protein blocks. Proc Natl Acad Sci USA. 1992;89(22):10915-9.

57. El-Gebali S, Mistry J, Bateman A, Eddy SR, Luciani A, Potter SC, Qureshi M, Richardson LJ, Salazar GA, Smart A, Sonnhammer ELL, Hirsh L, Paladin L, Piovesan D, Tosatto SCE, Finn RD. The Pfam protein families database in 2019. Nucleic Acids Res. 2019:47(D1):427-32.

58. Fabregat A, Jupe S, Matthews L, Sidiropoulos K, Gillespie M, Garapati P, Haw R, Jassal B, Korninger F, May B, Milacic M, Roca CD, Rothfels K, Sevilla C, Shamovsky V, Shorser S, Varusai T, Viteri G, Weiser J, Wu G, Stein L, Hermjakob H, D'Eustachio P. The Reactome pathway knowledgebase. Nucleic Acids Res. 2018;46(D1):649-55.

59. Carbon S, Ireland A, Mungall CJ, Shu S, Marshall B, Lewis S, Ireland A, Lomax J, Carbon S, Mungall C, Hitz B, Balakrishnan R, Dolan M, Wood V, Hong E, Gaudet P. AmiGO: online access to ontology and annotation data. Bioinformatics. 2009;25(2):288-9.

60. Bairoch A. The cellosaurus, a cell-line knowledge resource. J Biomol Tech. 2018;29(2):25-38.

61. Szklarczyk D, Franceschini A, Wyder S, Forslund K, Heller D, Huerta-Cepas J, Simonovic M, Roth A, Santos A, Tsafou KP, Kuhn M, Bork P, Jensen LJ, von Mering C. STRING v10: protein-protein interaction networks, integrated over the tree of life. Nucleic Acids Res. 2015;43(Database issue):447-52.

62. RC Team. R: A language and environment for statistical computing. Vienna, Austria: R Foundation for Statistical Computing; 2014

63. Ghose AK, Crippen GM. Atomic physicochemical parameters for three-dimensional structure-directed quantitative structure-activity relationships I. Partition coefficients as a measure of hydrophobicity. J Comput Chem. 1986:7(4):565-77.

64. Murdoch WJ, Singh C, Kumbier K, Abbasi-Asl R, Yu B. Definitions, methods, and applications in interpretable machine learning. Proc Natl Acad Sci USA. 2019;116(44):22071-80.

65. Tibshirani R. Regression shrinkage and selection via the lasso. J R Statl Soc Ser B. 1994;58:267-88.

66. Schwarz G. Estimating the dimension of a model. Ann Stat. 1978;6(2):461-4.

67. Gabriel F.R. HDeconometrics: implementation of several econometric models in high-dimension. R package version 2.10; 2016.

68. Buhlmann P, Van De Geer S. Statistics for high-dimensional data: methods, theory and applications. Berlin: Springer; 2011.

69. Zou H, Hastie T, Tibshirani R, et al. On the "degrees of freedom" of the lasso. Ann Stat. 2007;35(5):2173-92.

70. Witten IH, Frank E, Hall MA, Pal CJ. Data mining, Fourth edition: practical machine learning tools and techniques. 4th ed. San Francisco: Morgan Kaufmann Publishers Inc.; 2016.

71. Sall J, Stephens ML, Lehman A, Loring S. JMP start statistics: a guide to statistics and data analysis using JMP. Cary: Sas Institute; 2017.

72. Masters T. Practical neural network recipes in C++. San Diego: Academic Press Professional Inc; 1993.

73. Kelley CT. Iterative methods for optimization. Philadelphia: SIAM; 1999. 
74. Ng AY. Feature selection, 11 vs. 12 regularization, and rotational invariance. In: Proceedings of the twenty-first international conference on machine learning. ICML '04, p. 78. ACM, New York, NY, USA; 2004. https://doi. org/10.1145/1015330.1015435.

75. Blumer A, Ehrenfeucht A, Haussler D, Warmuth MK. Occam's razor. Inf Process Lett. 1987;24(6):377-80. https://doi. org/10.1016/0020-0190(87)90114-1.

76. Friedman J, Hastie T, Tibshirani R. Regularization paths for generalized linear models via coordinate descent. J Stat Softw. 2010;33(1):1-22.

77. Illuminating the Druggable Genome: Understudied proteins. https://commonfund.nih.gov/idg/understudiedpro teins (2019). Accessed 11 June 2019

78. Schrodinger L. The PyMOL molecular graphics system. Version. 2010;1(5).

\section{Publisher's Note}

Springer Nature remains neutral with regard to jurisdictional claims in published maps and institutional affiliations.

- fast, convenient online submission

- thorough peer review by experienced researchers in your field

- rapid publication on acceptance

- support for research data, including large and complex data types

- gold Open Access which fosters wider collaboration and increased citations

- maximum visibility for your research: over 100M website views per year

At BMC, research is always in progress.

Learn more biomedcentral.com/submissions 